J.R. Castrejon-Pita, S. Hoath and I Hutchings, Journal of Fluids Engineering, 134 (2012) 011201, 'Velocity Profiles in a Cylindrical Liquid Jet by Reconstructed Velocimetry' 


\section{Velocity profiles in a cylindrical liquid jet by reconstructed velocimetry}

\author{
J.R. Castrejón-Pita \\ Department of Engineering \\ University of Cambridge \\ 17 Charles Babbage Road \\ Cambridge CB3 OFS \\ United Kingdom \\ Email: jrc64@cam.ac.uk
}

\author{
S.D. Hoath \\ Department of Engineering \\ University of Cambridge \\ 17 Charles Babbage Road \\ Cambridge CB3 OFS \\ United Kingdom \\ Email: sdh35@cam.ac.uk
}

\author{
I.M. Hutchings \\ Department of Engineering \\ University of Cambridge \\ 17 Charles Babbage Road \\ Cambridge CB3 OFS \\ United Kingdom \\ Email: imh2@cam.ac.uk
}

\begin{abstract}
An experimental setup and a simple reconstruction method are presented to measure velocity fields inside nearly cylindrical liquid jets traveling through still air. Particle image velocimetry algorithms are used to calculate velocity fields from high speed images of jets of transparent liquid containing seed particles. An inner central plane is illuminated by a laser sheet pointed at the center of the jet and visualized through the jet by a high speed camera. Optical distortions produced by the shape of the jet and the difference between the refractive index of the fluid and the surrounding air are corrected by using a ray tracing method. The effect of the jet speed on the velocity fields is investigated at four jet speeds rates. The relaxation rate for the velocity profile downstream of the nozzle exit is reasonably consistent with theoretical expectations for the low Reynolds numbers and the fluid used, although the velocity profiles are considerably flatter than expected.
\end{abstract}

\author{
Nomenclature \\ $\rho$ Fluid density. \\ $\sigma$ Fluid surface tension. \\ $\mu$ Fluid viscosity
}

\section{Introduction}

The study of axi-symmetric jets of liquid flowing through still air is of great practical interest as liquid jets are created in many industrial applications where liquid prod- ucts or materials are delivered or transported. In particular, the reliability of some inkjet printing systems, rapid prototyping rigs, and material extrusion processes depends on the dynamic characteristics of liquids under the conditions found in these environments [1]. Although several techniques exist to study the dynamics of fluid flows, most are applicable only to systems with certain geometries and optical accessibility. Techniques such as laser Doppler anemometry (LDA), particle tracking velocimetry (PTV) and particle image velocimetry (PIV) are limited to the study of systems with planar surfaces and/or rectangular geometries. Conventional PTV and PIV methods rely on the analytical comparison of two sequential images to determine the motion of small particles within a fluid and hence the velocity of the fluid. The use of both techniques is limited by the framing speed or the time difference between consecutive images, the characteristics of the seeding particles, the field of view of the imaging camera and the thickness or depth of field of the visualization system, [2]. Conventional PIV typically relies on the use of specialized cameras to acquire two consecutive pictures with a frame separation such that the motion of the particles in the fluid can be tracked. For the correct application of PIV, the flow must be visualized without any kind of optical distortion in order to obtain velocity information from a well-defined region of the fluid [3], [4]. Optical distortions in a flowing fluid are commonly produced by differences between the refractive indexes of two liquids or between the liquid and its container, or between the liquid and the surrounding medium, [5] and [6]. As a consequence, PIV studies are often carried out in systems containing liquids with nearly 
matched refractive indices and in containers with parallel sides. These conditions limit the range of liquids that can be studied and restricts the geometrical properties of the systems used to transport or deliver them, [7]. A very recent approach is to correct the distortion produced by the differences in the refractive indices by using geometric optical principles such as ray-tracing. In particular, optical corrections to PIV analyzes have been successfully performed in order to image the flow structure inside an evaporating droplet deposited on hydrophilic substrates [8]. More recently, this methodology has also been theoretically expanded, but not tested, for hydrophobic substrates [9]. In a similar way, the experiments presented in this work demonstrate that a reconstruction approach can be used to obtain information about the velocity distribution within (tapered) cylindrical jets of liquids flowing in air.

In this paper, particle image velocimetry was used to measure the velocity profile across the central $90 \%$ of the width of a jet of a glycerol/water mixture emerging from a millimeter-scale nozzle. A simple correction method based on the ray-tracing method is presented to reconstruct and obtain flow details of the nearly cylindrical liquid jets at four different flow rates. At the fluid flow rates used in this work, high speed imaging was used to obtain the consecutive pictures required for PIV analysis. The conditions of the jets with the highest speeds, in terms of Reynolds and Weber number, match those typical of continuous ink-jet (CIJ) printing, and thus the results are directly applicable to that practical application. The methodology presented in this paper can be utilized on similar experimental scenarios where cylindrical jets flow in an optically different medium, i.e. flows from extrusion rigs, pipe flows and the fluid dynamics within cylindric tubes.

An incidental feature of the present work is that the nozzle exit face was always wetted by the fluid jet, so that the downstream jet profile was not pinned right at the edge of the nozzle outlet. Theoretical models for the velocity distributions in a continuous liquid jet generally assume an axisymmetric shape without such wetting, so that these direct PIV measurements also provide information which is not widely available from modeling.

\section{Experimental setup}

Experiments were performed in which a glycerol/water mixture was ejected from a $1.1 \mathrm{~mm}$ radius $\left(r_{0}\right)$ nozzle in a downward vertical direction to form stable jets with velocities ranging from 0.8 to $2.0 \mathrm{~m} / \mathrm{s}$. The design of this large scale model of an ink-jet printhead is described elsewhere [10]. The nozzle had an aspect ratio $L / D=2$ and a 1 $\mathrm{mm}$ long $45^{\circ}$ conical inlet section. This experimental system is easy to operate and provides a model environment which permits the use of conventional particle image velocimetry and other anemometry techniques. It is made of transparent polymer (poly-methyl methacrylate), operates at relatively slow average speeds $<5 \mathrm{~m} / \mathrm{s}$ and can jet liquids with viscosities ranging from $1 \mathrm{mPa}$ s to $100 \mathrm{mPa}$. The flow is regulated by a stabilized pump (Premotec BL58) and con-

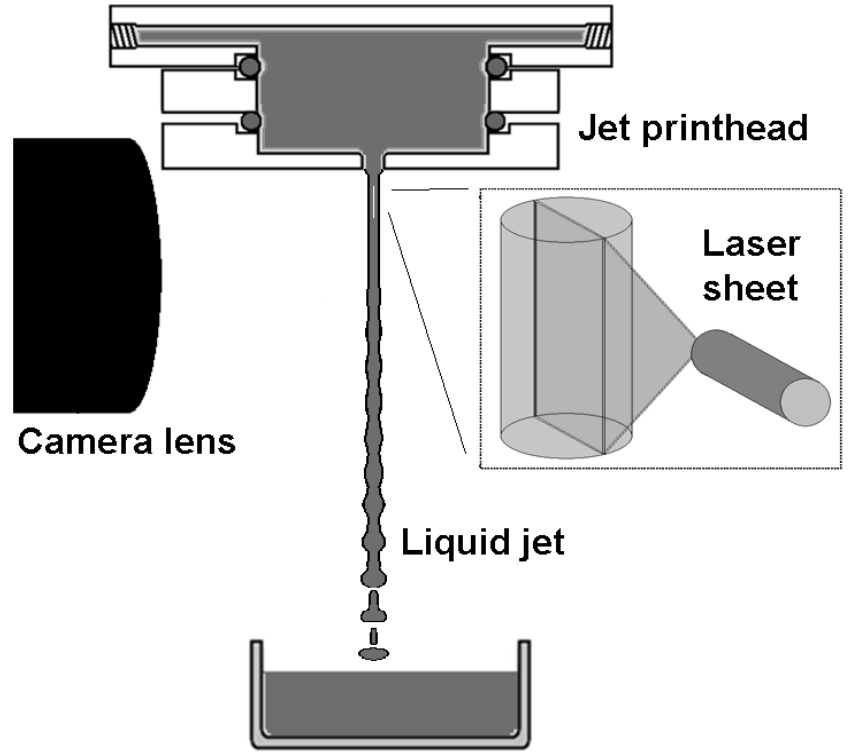

Fig. 1. Schematic view of the experimental setup.

tinuously measured with a turbine-type flow-meter (FHKSC Gigmesa) between the pump and the print-head. The Imaging setup consists of a Phantom V7.1 high speed camera with a Tamrom $90 \mathrm{~mm} \mathrm{~F} / 2.8$ macro lens set to produce a field of view of $5 \times 10 \mathrm{~mm}^{2}$ and a measured depth of field of $<0.5$ $\mathrm{mm}$. The camera was set up to image a rectangular region of interest parallel to the jet axis, of $256 \times 512$ pixels $^{2}$ (width $\times$ height). The magnification was measured to correspond to $0.0213 \mathrm{~mm} / \mathrm{pixel}$ from images of a ruled scale. Images were captured at an interval of $111.0 \mu$ s with an exposure time of $60 \mu \mathrm{s}$. The illumination was provided by a continuous laser diode (532 nm wavelength, $200 \mathrm{~mW}$ power) through a 2.2 $\mathrm{mm}$ diameter glass cylindrical lens to produce the laser sheet. In addition, an inverted beam expander (10 to 1 magnification) was used to narrow the laser beam. With this optical system a laser sheet with a waist of $<200 \mu \mathrm{m}$ was produced and aligned parallel to the jet axis and towards the center of the jet as shown in Fig. 1. The axis of the high speed camera was positioned perpendicular to the laser sheet and aligned in such a way that the jet boundaries appeared sharply focused.

The Newtonian liquid used in all the experiments was a mixture of $74.7 \%$ glycerol (99.9\% pure) with $25.3 \%$ tridistilled water (weight percentage). Its measured properties were: density $\rho=1250 \mathrm{~kg} / \mathrm{m}^{3}$; surface tension $\sigma=65 \mathrm{mN} / \mathrm{m}$ (measured with a bubble tensiometer: SITA Messtechnik, Pro line t15, at a bubble lifetime of $100 \mathrm{~ms}$ ); viscosity $\mu$ $=36.5 \mathrm{mPa}$ s (measured with a vibrational viscometer: Hydramotion Visco-lite 700).

The refractive index $n$ for the liquid-air interface was taken to be 1.435. Seeding particles of titanium dioxide $\left(\mathrm{TiO}_{2}\right) \approx 2 \mu \mathrm{m}$ in diameter were suspended in the liquid by stirring, and ultrasonic mixing. Typical images are shown in Fig. 2 in which the particles are readily distinguished. Four terminal average jet speeds $(v=0.59,1.00,1.61$ and 2.27 $\mathrm{m} / \mathrm{s}$ ) were used for these experiments. In terms of dimensionless numbers and the jet terminal average speed $v$, the 

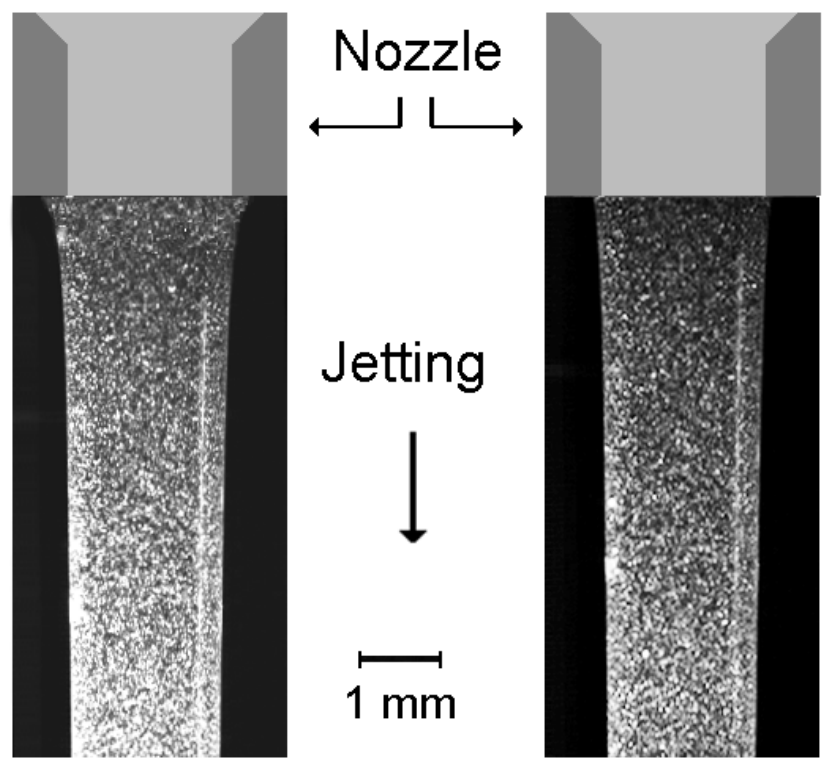

Fig. 2. (Colour online) High speed images of a $0.59 \mathrm{~m} / \mathrm{s}$ fluid jet (left image) and a $2.27 \mathrm{~m} / \mathrm{s}$ fluid jet (right image) both captured with an exposure time of $60 \mu \mathrm{s}$. The jets contain small particles and taper downstream. The liquid in the slower jet wets the nozzle exit face significantly as it extends to $\approx 140 \%$ of the nozzle diameter. At higher speeds, the jet also tapers downstream but there is less extensive wetting at the nozzle.

Reynolds and Weber numbers are defined as

$$
\operatorname{Re}=\frac{\rho v r_{0}}{\mu}, \quad \mathrm{We}=\frac{\rho v^{2} r_{0}}{\sigma}
$$

Under the flow conditions of these experiments, four dimensionless combinations were studied: $i$ ) $\mathrm{Re}=22.2$ and $\mathrm{We}=$ 7.4, ii) $\mathrm{Re}=37.7$ and $\mathrm{We}=21.2$, iii) $\mathrm{Re}=60.7$ and $\mathrm{We}=54.9$ and $i v) \mathrm{Re}=85.5$ and $\mathrm{We}=109.0$. Only the jet region closer than $10 \mathrm{~mm}$ to the nozzle exit (i.e. within about 4.5 nozzle diameters) is of interest here; beyond this, gravity and instabilities affect the jet speed and profile [11].

The presence of nozzle face wetting means that the jet tapers along its length, although it has been long known that even jets pinned at the nozzle exhibit diameter changes downstream [12]. At the speeds used here, the jet diameter tapers gradually after a more rapid initial rate of diameter change which follows a one-third power law in distance $z$, [13]. The shape of the jets as a function of distance $z$ were parameterized at each jet speed, so that the ray-tracing corrections discussed below could be applied.

\section{Axial reconstruction}

Figure 3 shows the geometry and variables used in the reconstruction. The nozzle face is at distance $z=0$ and the jet direction is downwards in the negative z-direction: the PIV reconstruction only involves those regions that were accessible to the technique, $z \leq-0.25 \mathrm{~mm}$. The wetted nozzle face, the tapering of the jet radius downstream of the nozzle

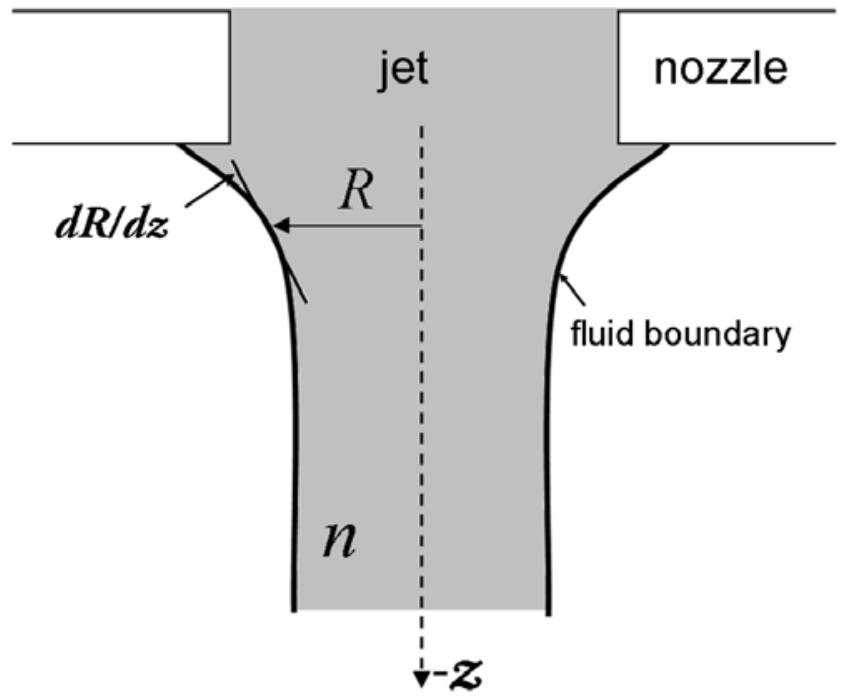

Fig. 3. (Colour online) Diagram showing the variables relevant to the axial reconstruction.

exit and the final jet diameter are represented by the curved, axi-symmetric, fluid boundary with radius $R$ and (positive) slope $d R / d z$ at $z$. The jet refractive index is $n$ and ambient gas is assumed to have a refractive index of unity

Two PIV algorithms, MATPIV and URAPIV, were used in this work with consistent results, [14] and [15]. In the analysis, the region surrounding the jet area was masked with a rectangular mask with a width corresponding to the downstream jet diameter and a length corresponding to the distance of the jet from the nozzle exit to the end of the image. PIV analyzes were performed on interrogation areas of $8 \mathrm{x}$ 16 pixels $\left(85 \times 170 \mu \mathrm{m}^{2}\right)$ with horizontal and vertical spacings of 4 and 8 pixels respectively. For the geometry and physical nature of the flows studied here, it was found that the axial component of the velocity was consistently two order of magnitude greater than the radial component which is the one more affected by optical distortion by light refraction through the transparent jet. Correction algorithms as described below were applied to amend the position of the interrogation areas (and hence the velocity vectors) but were not used to correct the position of individual particles on the image.

In a typical PIV algorithm, an image is divided into interrogation areas in which the motion of particles is analyzed, [3]. In the present case, the positions of these interrogation areas will be affected by both the cylindrical radius of curvature of the jet and by the tapering curvature of $d R / d z$ due to the wetting of the nozzle plane, the relaxation of the jet diameter and the gravitational acceleration of the fluid [13]. Figure 4 shows the methodology followed during the reconstruction process. The true position of these interrogation areas depends on the refractive index of the liquid medium and the local radius of curvature of the jet. By considering a ray entering the camera orthogonally to the $z$ axis of the jet (perpendicular to the plane shown in Fig. 4), from an object 
at $z_{o b j}$, it can be shown that

$$
z_{o b j} \approx z+R \tan \left[\frac{n-1}{n} \frac{d R}{d z}\right],
$$

where $n$ is the refractive index of the liquid, $R$ is the true jet radius and $\mathrm{d} R / \mathrm{d} z$ is the axial slope at axial image position $z$ downstream of the nozzle exit. The axial slope $d R / d z$ is positive for the axial jet profile, so that $z_{o b j} \geq z$.

The axial change of jet curvature over the length scales involved in the PIV measurement produces significant axial positional errors only for $z \geq-0.25 \mathrm{~mm}$ from the nozzle exit. Therefore the experimentally observed $z$ values can be used to quantify the actual jet radius $R$ along the jet, with axial errors of $\pm 0.01 \mathrm{~mm}$, which are used later when discussing the radial reconstruction.

Table 1. Jet exit and downstream radii $(\mathrm{mm})$.

\begin{tabular}{lcccc}
$v(\mathrm{~m} / \mathrm{s}):$ & 0.59 & 1.00 & 1.61 & 2.27 \\
\hline \hline At nozzle exit: & 1.55 & 1.48 & 1.38 & 1.31 \\
Measured $\left(\mathrm{z}=8 r_{0}\right):$ & 1.01 & 1.01 & 1.00 & 1.01 \\
Predicted $\left(\mathrm{z}=8 r_{0}\right):$ & 0.97 & 0.98 & 0.99 & 1.00 \\
\hline
\end{tabular}

Table 1 gives jet diameters, for the four jet speeds in the present work, as actually measured at the exit and at $z=8 r_{0}$ downstream, and also the predicted downstream radii at $z=8 r_{0}$ for un-wetted CIJ nozzles, [12]. Predictions using the conservation of mass and momentum for horizontal Newtonian jets suggest minimum value for the ratio of radii of final jet to the nozzle is $\chi=\sqrt{3} / 2$. Fuller treatment showed that $\chi$ is a function of the Reynolds and Weber numbers [12]. This analytic treatment has been used to provide the values in Table 1, for the condition of our experiments after allowance for corrections to results given in [12]. The thinning effects of gravity can be ignored for our restricted measurement range

The small $(<4 \%)$ differences between the measured downstream radii $R$ and the radii predicted for an un-wetted nozzle may be due to the nozzle wetting; the jet at the lowest speed shows the largest wetted radius $(\approx 140 \%$ of the nozzle radius) and the largest radial excess $(\approx 4 \%)$ over the value predicted for the un-wetted nozzle, while the jet at the highest speed showed the lowest wetted radius and almost the same radius at $z=8 r_{0}$ downstream.

After establishing that the fluid jet beyond the nozzle region is almost cylindrical, and also with a diameter close to the predicted values despite the wetting of the nozzle exit, the main focus of the present work is then the radial reconstruction of the velocity profile.

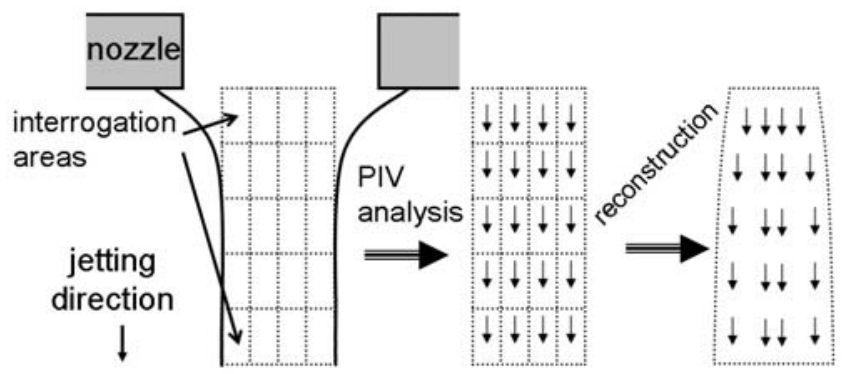

Fig. 4. (Colour online) Diagram showing the methodology followed during the reconstruction process.

\section{Radial reconstruction}

Optical distortion in the radial direction does not occur for an object (or an interrogation area) lying on the central $z$ axis or at the outer edges of the jet. Between these extremes, the radial coordinate $r_{o b j}$ for an object would be recorded at image radius $r$ due to refraction through a liquid lens of refractive index $n$ and true radius $R$ at axial position $z$ downstream of the nozzle exit. In the present experiments, the objects of interest lie in the area illuminated by the laser sheet and thus in the central plane of the jet. Trigonometry and Snell's law of refraction at the fluid-air interface can be used to derive the radial correction algorithm for a cylindrical jet, which gives:

$$
r_{o b j}=r-\sqrt{R^{2}-r^{2}} \tan \left[\arcsin \frac{r}{R}-\arcsin \frac{r}{n R}\right] .
$$

Figures 5 to 8 show the PIV results as contour plots of the instantaneous velocity field for the four jet speeds used in the experiments. In each case the left image shows the radially distorted velocity field and the right one shows the radially corrected field. The downstream image radii in each case A) appear to be very close to $1.0 \mathrm{~mm}$, as given in Table 1 and determined directly from photographs of the jets (such as that shown in Fig. 2). Figures 5 and 6, the most wetted cases, show how interrogation areas placed at both extremes of the region of interest are differently affected depending on their positions along the jet. The necking of the jet causes that the upper PIV interrogation areas are centred on a jet with wider diameter than the lower areas that span the width of the jet. For each case B) the downstream radial extent is still $\simeq 1.0 \mathrm{~mm}$, while the radial reconstruction of the PIV results by using equation (2) significantly changes the spacing of the velocity vectors, such that the outermost spacing is much larger than the innermost spacing, by a factor of $\approx n$.

In all these cases, local velocities within the field do not extend as far as would be expected for fully developed parabolic velocity profiles across the nozzle exit, i.e. up to twice the average speed of the jet. This is especially marked for the lowest jet speed, which for fully developed flow would be expected to peak close to $1.2 \mathrm{~m} / \mathrm{s}$, but is also clear for the fastest jet at $2.27 \mathrm{~m} / \mathrm{s}$, which would peak at 4.5 $\mathrm{m} / \mathrm{s}$.

Figures 9 to 12 show results of the radial reconstruction or the reconstructed velocity profiles at different jet ax- 


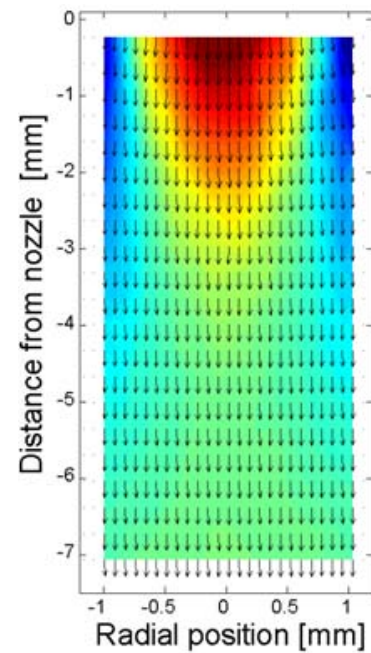

A)

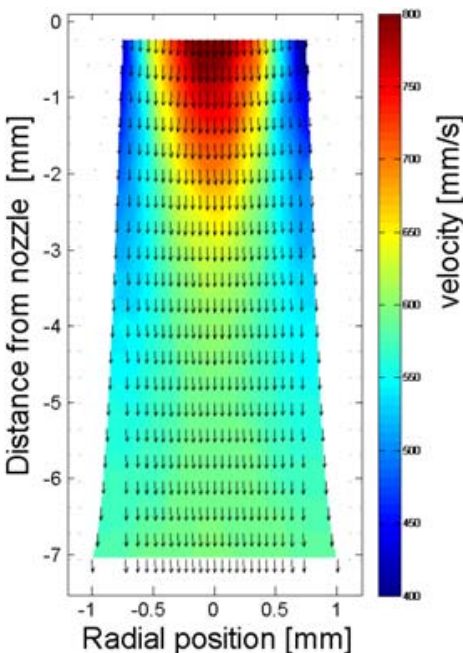

B)

Fig. 5. (Colour online) Instantaneous velocity field inside a liquid jet with a terminal speed of $0.59 \mathrm{~m} / \mathrm{s}$ : A) radially distorted velocity field; B) corrected field.

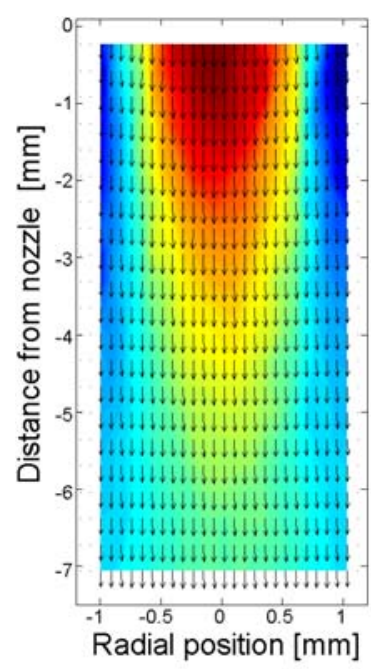

A)

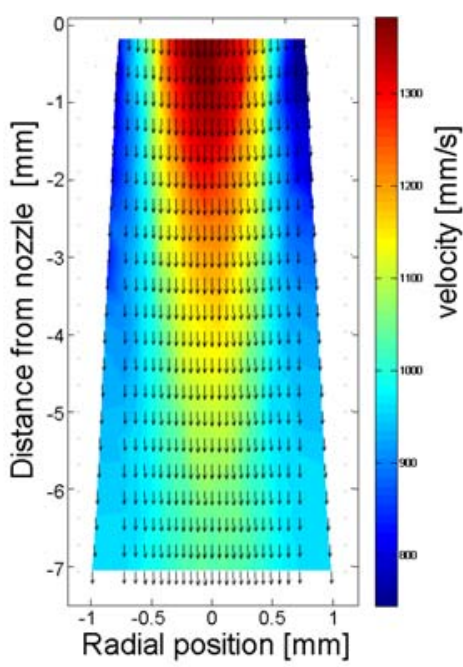

B)

Fig. 6. (Colour online) Instantaneous velocity field inside a liquid jet with a terminal speed of $1.00 \mathrm{~m} / \mathrm{s}$ : A) radially distorted velocity field; B) corrected field.

ial positions $z=-1,-2,-3,-4,-5,-6$, and $-7 \mathrm{~mm}$ downstream from the nozzle. Data points which were too close to the jet edges for reliable analysis or interpretation have been excluded from the plots.

\section{Velocity profile relaxation}

Relaxation of the strong variation of the velocity with radial position, from a fully developed parabolic velocity profile within the nozzle into a "flat" or "plug" profile independent of radial position occurs downstream of the nozzle once the wall stresses are no longer physically present on the jet to impede equalisation of the fluid profile. The initial velocity profile need not be fully developed in short nozzles,

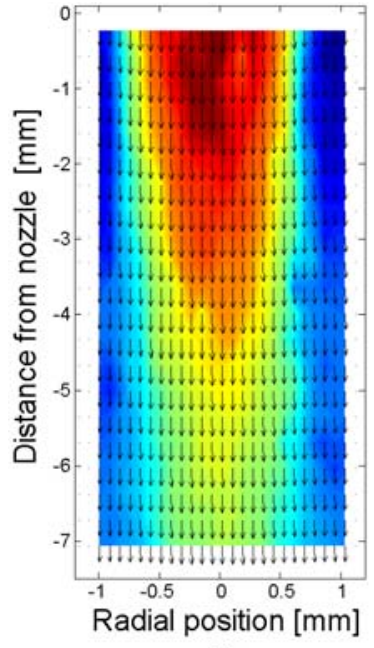

A)

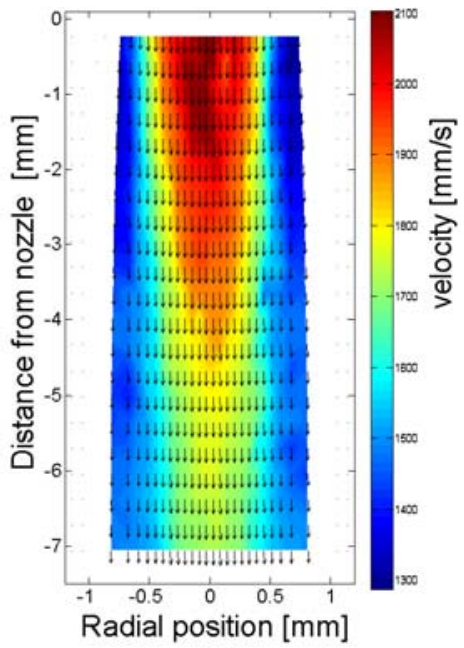

B)
Fig. 7. (Colour online) Instantaneous velocity field inside a liquid jet with a terminal speed of $1.61 \mathrm{~m} / \mathrm{s}$ : A) radially distorted velocity field; B) corrected field.

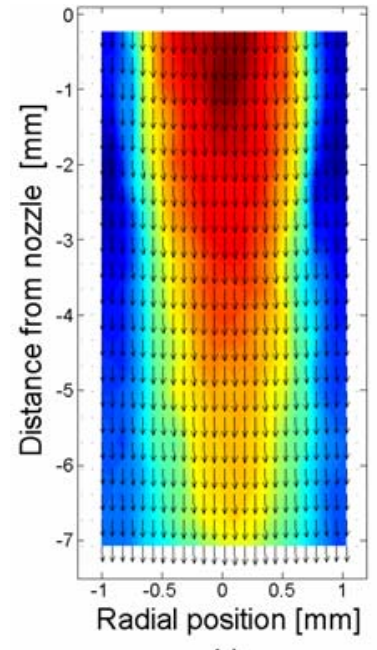

A)

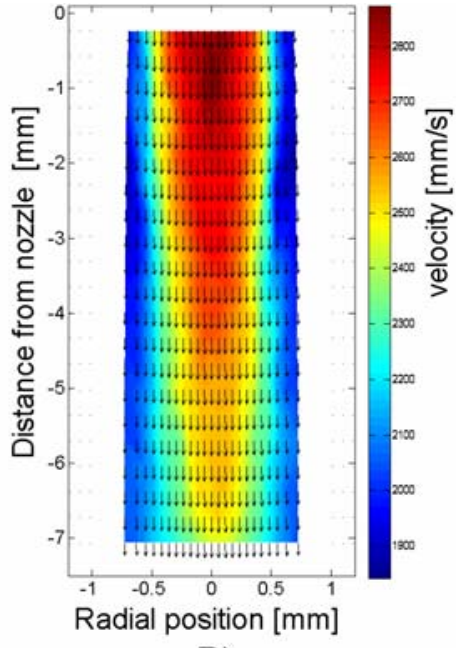

B)
Fig. 8. (Colour online) Instantaneous velocity field inside a liquid jet with a terminal speed of $2.27 \mathrm{~m} / \mathrm{s}$ : A) radially distorted velocity field; B) corrected field.

as shown in [16], and others, but the fluid viscosity acts to equalise the free jet profile. The relaxation of the velocity profile initially depends on the initial conditions at the nozzle exit, which may depend on the degree of nozzle exit face wetting by the fluid, but soon develops a single characteristic rate determined by the cylindrical geometry, as involving the smallest non-zero root of a Bessel function, according to [17].

Theoretical computations for the velocity profile expected at the end of a straight tube of finite length, with no wetting at the nozzle exit have been derived previously [16]. Formulas for the velocity profile relaxation downstream from a long circular nozzle have also been derived previously $[13,18]$. The analytical solutions derived for this case include summations over exponential terms with decay 


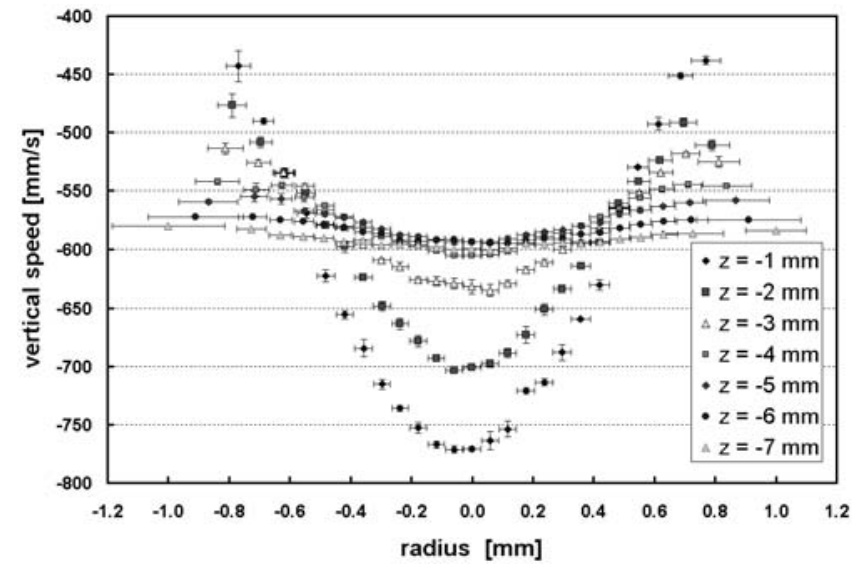

Fig. 9. (Colour online) Corrected velocity profiles for jet at $0.59 \mathrm{~m} / \mathrm{s}$ from a wetted nozzle.

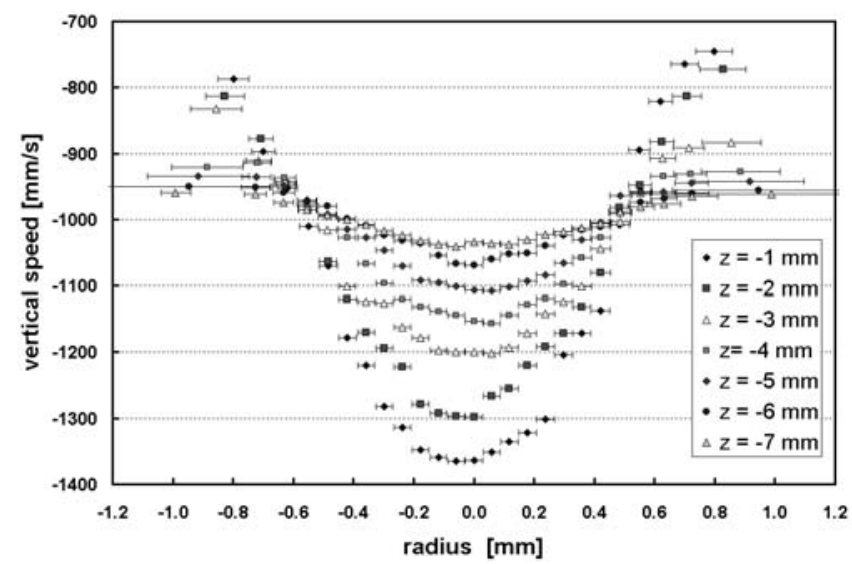

Fig. 10. (Colour online) Corrected velocity profiles for jet at 1.00 $\mathrm{m} / \mathrm{s}$ from a wetted nozzle.

constants determined by the square of the positive roots of the Bessel function $J_{1}(x)$ divided by the Reynolds number Re. The persistent decaying contribution therefore arises from $x_{1}=3.8317 \ldots$, the smallest non-zero root of $J_{1}(x)$. Furthermore, this persistent decaying contribution is significant even in the nozzle exit region in order that the velocity profile there avoids a change of the sign in $\mathrm{d} u / \mathrm{d} r$ between the centre and the edge of the jet [17]. For the present experiments at low jet speeds the velocity profiles would be expected to be close to parabolic for an un-wetted nozzle exit. Downstream from the nozzle the liquid undergoes changes in both its diameter and velocity profile changes but rather slowly attains a flat velocity profile. The difference between the centre and the outer edge velocities at axial position $z$, normalized by the average velocity, can be defined as the velocity profile range at $z$ (and equals 2 for parabolic flow). The persistent exponential decay constant $\lambda$ of the velocity profile range with dimensionless distance $z / r_{0}$ depends on the fluid properties, the nozzle radius $r_{0}$ and average fluid speed $v$, via the inverse of the Reynolds number. The theoretical value for the persistent exponential decay constant is $\lambda=x_{1}^{2} / \mathrm{Re}$ in dimensionless units, [18].

Comparison between the decay constants for the speed profile with the theory [16] and the decay constant $\lambda$ can

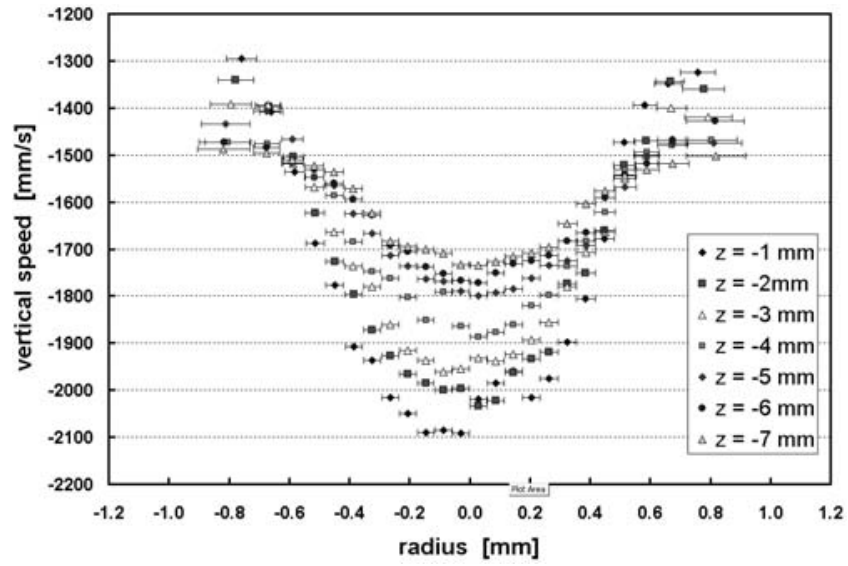

Fig. 11. (Colour online) Corrected velocity profiles for jet at 1.61 $\mathrm{m} / \mathrm{s}$ from a wetted nozzle.

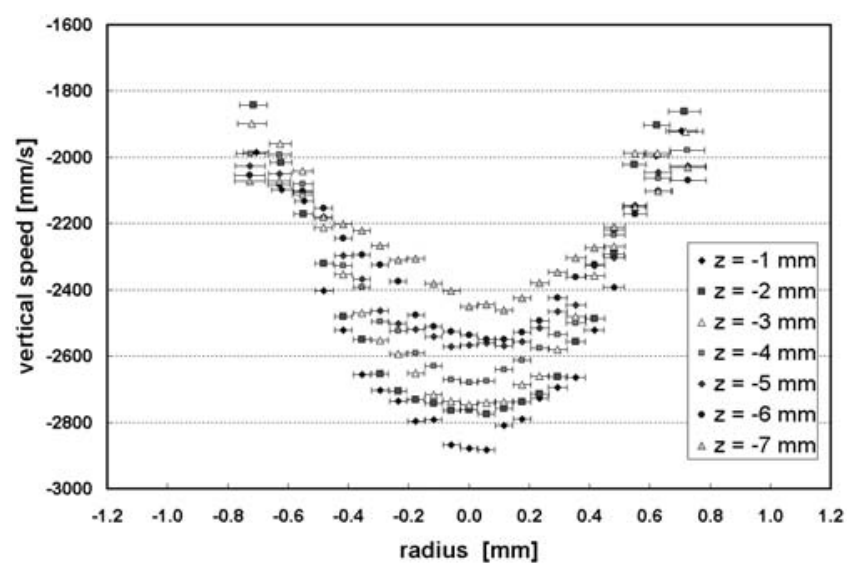

Fig. 12. (Colour online) Corrected velocity profiles for jet at 2.27 $\mathrm{m} / \mathrm{s}$ from a wetted nozzle.

be deduced from the PIV experiments. Figures 13 to 16 show the dimensionless range of velocity (the difference between the axial and outer speeds divided by the radial average jet speed), plotted on a logarithmic scale as a function of the downstream distance in $\mathrm{mm}$. A flattened initial decay is followed by a steady exponential decay as predicted by the theory, [17], but eventually limited by resolution beyond $\sim z<-6 \mathrm{~mm}$. Lines of best fit are superimposed on the graphs.

Table 2 lists, the decay constants for the exponential fit to the speed profile obtained from the PIV data 2 to $6 \mathrm{~mm}$ downstream from the nozzle exit and for comparison, theoretical decay constants for un-wetted nozzles expressed in $\mathrm{mm}^{-1},[18]$.

As expected, Table 2 shows the decay constant for the fluid downstream of the nozzle exit to be independent of the extent of nozzle wetting. The decay constant is found to be $0.34 / \mathrm{speed}(\mathrm{m} / \mathrm{s})$ for distance $z$ in units of nozzle radius $r_{0}$, close to that the value of $0.39 / \mathrm{speed}(\mathrm{m} / \mathrm{s})$ deduced from theoretical considerations for this fluid $[17,18]$. This close correspondence suggests that the reconstructed velocimetry values are probably correct to within better than $15 \%$ accuracy. 


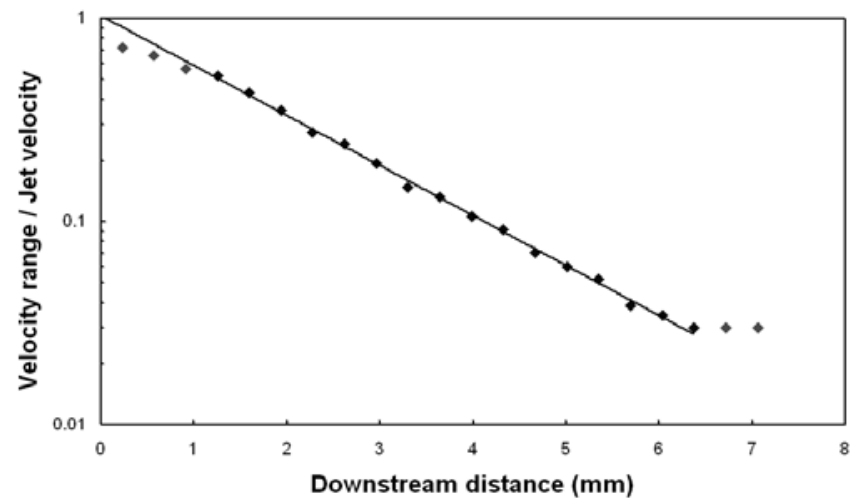

Fig. 13. (Colour online) Velocity range plotted against downstream distance $(\mathrm{mm})$ for $0.59 \mathrm{~m} / \mathrm{s}$ jet from a wetted nozzle.

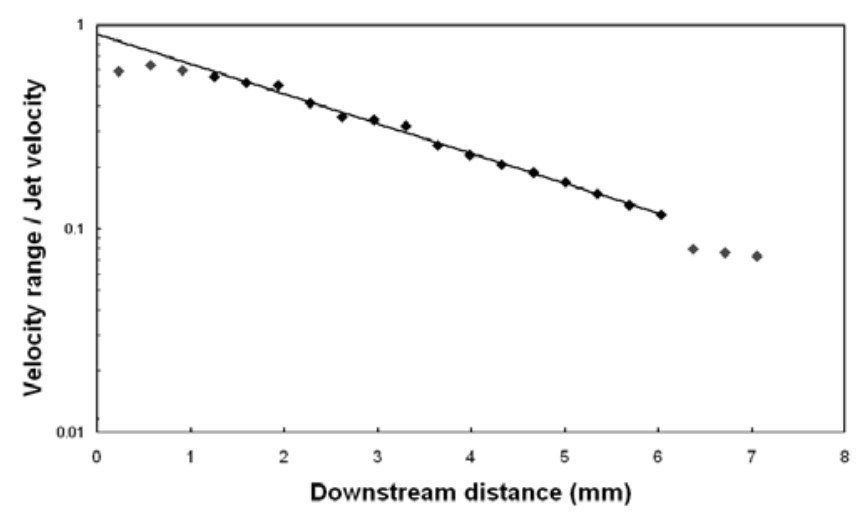

Fig. 14. (Colour online) Velocity range plotted against downstream distance $(\mathrm{mm})$ for $1.00 \mathrm{~m} / \mathrm{s}$ jet from a wetted nozzle.

\section{Wetted nozzles}

The main value of the PIV measurements was not to confirm predictions based on analytical solutions from fluid dynamics theory (or numerical simulations) for un-wetted nozzles, but to explore the effects of nozzle wetting on the velocity profiles. It is well-known that nozzle face wetting slightly enlarges the jet diameter for some distance downstream from the exit, which must expected to alter the radial velocity distribution somewhat from that predicted by the approximate analytical solutions [18] (or simulation results [19]) for un-wetted nozzles. Figures 13-16 all show that as the jet leaves the wetted nozzle, the normalized velocity range is significantly less than 1 , rather than close to the value of 2 expected for fully developed flow from an unwetted nozzle. This is even true for the lowest jet speed, for which the flow should be nearly $98 \%$ developed in un-wetted nozzles with the geometry used in the present experiments.

The region closer to the nozzle face will be influenced by dissipative effects of the fluid viscosity, the non-parabolic velocity profile and the nozzle face wetting, and also by radial flows ignored by the simple model, which is valid for $300<\operatorname{Re}<1000$, [20]. Simple predictions from a more rigorous treatment [18] are also limited to the regime $200<\operatorname{Re}$ $<1000$, whereas in our experiments $40<\operatorname{Re}<180$. Our expectations are that as the fluid viscosity used $(36.5 \mathrm{mPa} \mathrm{s})$ is significantly higher than that of water $(1 \mathrm{mPa} \mathrm{s})$, non-linear

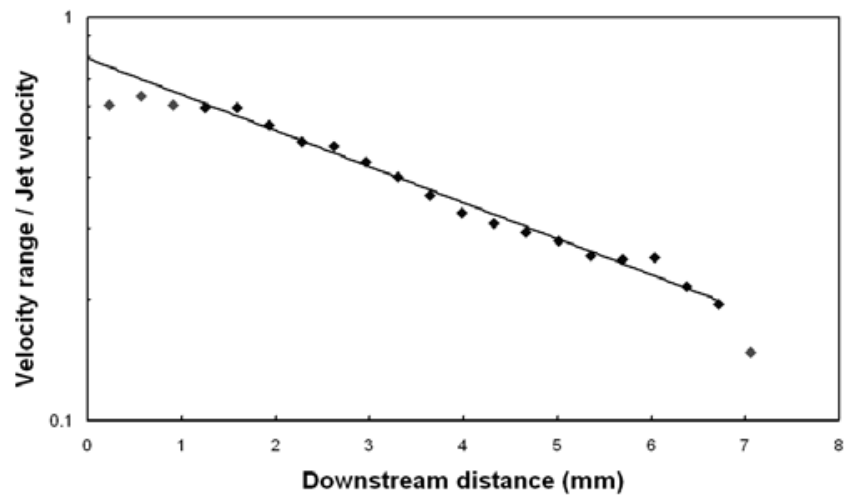

Fig. 15. (Colour online) Velocity range plotted against downstream distance $(\mathrm{mm})$ for $1.61 \mathrm{~m} / \mathrm{s}$ jet from a wetted nozzle.

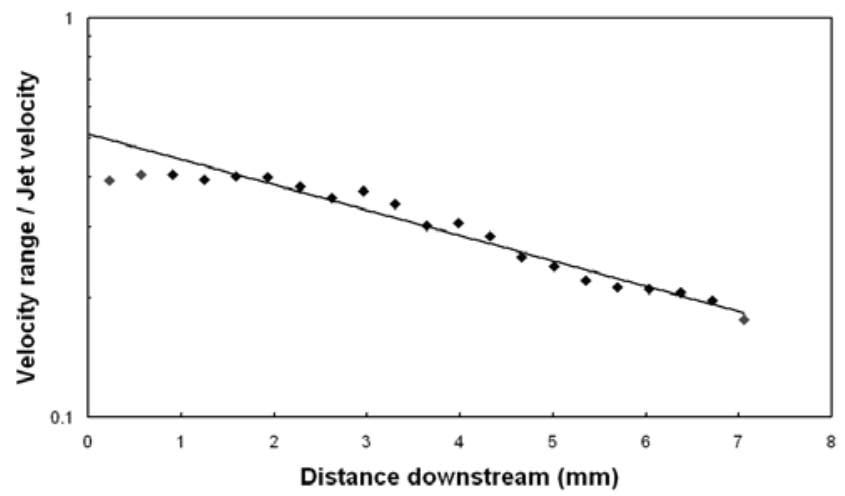

Fig. 16. (Colour online) Velocity range plotted against downstream distance $(\mathrm{mm})$ for $2.27 \mathrm{~m} / \mathrm{s}$ jet from a wetted nozzle.

fluid dynamics must be considered close to the wetted nozzle (subject to mass conservation). In particular, the usual boundary condition on the jet at the nozzle exit must be seriously compromised. The net result of nozzle wetting may be to damp any nozzle perturbations.

Table 2. Decay constants.

\begin{tabular}{lcccc}
$v(\mathrm{~m} / \mathrm{s}):$ & 0.59 & 1.00 & 1.61 & 2.27 \\
\hline \hline PIV $\left(\mathrm{mm}^{-1}\right):$ & 0.57 & 0.34 & 0.21 & 0.15 \\
Theory $\left(\mathrm{mm}^{-1}\right):$ & 0.66 & 0.39 & 0.24 & 0.17 \\
\hline
\end{tabular}

\section{Conclusions}

High speed imaging has been used to record the flow within continuous jets of liquid emerging from a circular nozzle under conditions which match those typical of continuous ink-jet printing. PIV analysis of $2.2 \mathrm{~mm}$ diameter jets in air was presented. Internal velocity fields were obtained by PIV and were corrected for optical refraction within the tapering liquid column by a simple correction method based on 
geometrical optics and Snell's law. The experimental results are consistent with previous experiments and in agreement with established theories for fluid flow. However, the PIV results also showed that nozzle wetting can significantly influence the magnitude of the velocity range at the nozzle exit, presumably because the usual boundary condition on the jet size (and radial velocity) at the nozzle exit is not appropriate immediately beyond it.

\section{Achnowledgements}

This work was supported by the Engineering and Physical Sciences Research Council (UK) and by industrial partners in the Innovation in Industrial Inkjet Technology (I4T) project.

\section{References}

[1] Frohn, A., and Roth, N., 2000, Dynamics of Droplets, Springer Verlag, Berlin, Germany, Chap. 1.

[2] Olsen, M. G., and Bourdon, C. J., 2003, "Out-of-Plane Motion Effects in Microscopic Particle Image Velocimetry”, J. Fluid Eng., 125, pp. 895-901.

[3] Adrian, R.J., 1991, "Particle-imaging techniques for experimental fluid mechanics", Annu. Rev. Fluid Mech., 23, pp. 261-304.

[4] Meinhart, C. D., Wereley, S. T., and Santiago, J. G., 2000, "A PIV Algorithm for Estimating Time-Averaged Velocity Fields", J. Fluid Eng., 112, pp. 285-289.

[5] Kaneda, M., Hyakuta, k., Takao, Y., Ishizuka, H., and Fukai, J., 2008, "Internal flow in polymer solution drolets deposited on a lyphobic surface during a receding process", Langmuir, 24, pp. 9102-9109.

[6] Parker, J., and Merati, P., 1996, "An Investigation of Turbulent Taylor-Couette Flow Using Laser Doppler Velocimetry in a Refractive Index Matched Facility”, J. Fluid Eng., 118(4), pp. 810-819.

[7] Castrejón-Pita, J. R., Betton, E. S., Kubiak, J. K., Wilson, 2011, M. C. T., and Hutchings, I. M., "The dynamics of the impact and coalescence of droplets on a solid surface", Biomicrofluidics, 5, Paper No. 014112.

[8] Kang, K. H., Lee, S. J., Lee, C. M., and Kang, I. S., 2004, "Quantitative visualization of flow inside an evaporating droplet using the ray tracing method", Meas. Sci. Technol., 15, pp. 1104-1112.

[9] Minor, G., Oshkai, P., and Djilali, N., 2007, “Optical distortion correction for liquid droplet visualization using the ray tracing method: further considerations" Meas. Sci. Technol., 18, pp. L23-L28.

[10] Castrejón-Pita, J.R., Martin, G. D., Hoath, S. D., and Hutchings, I. M., 2008, “A simple large-scale droplet generator for studies of inkjet printing”, Rev. Sci. Instrm., 79, Paper No. 075108.

[11] Lopez, B., Soucemarianadin, A., and Attané, P., 1999, "Break-up of continuous liquid jets: effect of nozzle geometry", J. Imaging Sci. Technol., 43(2) pp.145-152.

[12] Sarkar, K., and Schowalter, W. R, 2002, "Computation of a free jet with embedded drops", J. Non-Newtonian Fluid Mech. 102 pp. 263-280.

[13] German, R., and Khayat, R. E., 2005, "Steady and transient thin-jet flow", Physics of Fluids, 17, Paper No. 102104.

[14] Taylor, Z., Gurka, R., Liberzon, A., and Kropp, G., 2008, "Open source PIV software applied to streaming, time-resolved PIV data", Proc. 61st Annual Meeting of the Division of Fluid Dynamics of the American Physical Society, San Antonio, Texas.

[15] J. K. Sveen, 2004, An Introduction to MatPIV v.1.6.1, Department of Mathematics, University of Oslo, Oslo, Norway, ISSN 0809-4403.

[16] Durst, F., Ünsal, B., and Bayoumi, O. A., 2005, "The Development Lengths of Laminar Pipe and Channel Flows", J. Fluids Engineering, 127, pp. 1154-1160.

[17] González, H., and García, F. J., 2009, "The measurement of growth rates in capillary jets", J. Fluid Mechanics, 619, pp. 179-212.

[18] Pan, Y., and Suga, K., 2006, "A numerical study on the breakup process of laminar liquid jets into a gas", Physics of fluids, 18, Paper No. 052101.

[19] Morrison, N. F, and Harlen, O. G., 2010, "Viscoelasticity in inkjet printing", Rheologica Acta, 49, pp. 619-632.

[20] González-Mendizabal, D., Olivera-Fuentes, C., and Guzmán, J. M., 1987, "Hydrodynamics of laminar liquid jets. Experimental study and comparison with two models", Chem. Eng. Comm., 56, pp. 117-137. 